\title{
CARACTERÍSTICAS DE GESTIÓN, CONTROLES GERENCIALES Y DE RENDIMIENTO DE EMPRESAS MYPES
}

\author{
MANAGEMENT CHARACTERISTICS, MANAGERIAL \\ AND BUSINESS PERFORMANCE CONTROLS
}

\author{
Felipe André Della Giustina* \\ Graduado de Ciencias Contables \\ lipeufsc2017@hotmail.com \\ Edicreia Andrade dos Santos* \\ Doutranda en Contabilidad \\ edicreiaandrade@yahoo.com.br \\ Rogério João Lunkes* \\ Graduado de Ciencias Contable \\ Profesor y doctor en Contabilidad \\ rogerio.lunkes@ufsc.br \\ Fabricia Silva da Rosa* \\ Profesor y doctor en Contabilidad \\ fabriciasrosa@hotmail.com \\ Guilherme Bertamoni* \\ Graduado de Ciencias Contables \\ gui.bertamoni@gmail.com \\ *Docentes de la Universidad Federal de Santa Catarina
}

\section{Resumen}

En este estudio se evidencia la importancia del uso de los controles gerenciales como herramientas de gestión contable-financiera de modo que permitan orientar a los empresarios de las micro y pequeñas empresas a mantenerse en el mercado de forma competitiva. El estudio tiene como objetivo verificar las características de gestión, controles gerenciales y de desempeño de las empresas MyPES del rubro alimenticio. Se realizó una investigación por encuestas con los gestores, en 31 empresas del rubro alimenticio, como bares y restaurantes de la ciudad de Florianópolis, Santa Catarina. En el análisis de los datos se utilizó estadística descriptiva. Los resultados evidenciaron que la mayoría de las empresas utilizan los controles gerenciales como herramientas de gestión y control del rendimiento, 
para que puedan colocar en práctica sus acciones, buscando alcanzar sus objetivos. El uso de los controles gerenciales en todas las esferas de la empresa contribuye al buen desempeño de la organización; sin embargo, el estudio reveló también, que algunas empresas aún necesitan ayuda en relación al uso de los controles gerenciales para dar un norte a sus acciones y alinear sus objetivos.

Palabras Clave: Control gerencial, rendimiento, alimentación, MyPES.

\begin{abstract}
This study shows the importance of the use of management controls as an accounting and financial management tool to guide entrepreneurs in micro and small companies to remain competitive in the market. The study aims to verify the management characteristics, management and performance controls of food business SMEs. A survey was conducted with managers in 31 food companies, such as bars and restaurants in the City of Florianópolis, Santa Catarina (Brazil). Data were analyzed using descriptive statistics. The results showed that most companies use management controls as tools of management and performance control, so that they can put their actions into practice, aiming at reaching the objectives. The use of management controls in all spheres of the company contributes to the good performance of the organization, however, the study also revealedthat some companies still need help regarding the use of management controls to guide their actions and thus align their objectives.
\end{abstract}

Keywords: Management control, Performance, Food, SMEs.

\title{
Resumo
}

Neste estudo é evidenciada a importância do uso dos controles gerenciais como ferramenta de gestão contábil-financeira para orientar os empresários de micro e pequenas empresas a se manterem no mercado de forma competitiva. O estudo tem como objetivo verificar as características de gestão, controles gerenciais e de desempenho de empresas MPEs do ramo alimentício. Uma pesquisa de levantamento foi realizada com gestores, em 31 empresas do ramo alimentício, como bares e restaurantes da Cidade de Florianópolis, Santa Catarina. Na análise dos dados utilizou-se estatística descritiva. Os resultados evidenciaram que a maioria das empresas utilizam os controles gerenciais como ferramentas de gestão e controle de desempenho, para que possam colocar em pratica suas ações, visando o alcance dos objetivos. A utilização dos controles gerenciais em todas as esferas da empresa contribui para o bom desempenho da organização, porém, o estudo revelou, também que algumas empresas ainda precisam de auxilio com relação ao uso dos controles gerenciais para nortear suas ações e alinhar assim seus objetivos.

Palavras-chave: Controle gerencial, Desempenho, Alimentício, MPES. 


\section{Introducción}

Desde el inicio de la civilización, la contabilidad ha estado presente, mostrándose vital desde las primeras manifestaciones humanas y en el instante en que el hombre necesitó administrar sus posesiones. Según Villa (2012), la sociedad tiene necesidad de ayuda para obtener los datos que busca, «dado que su diversidad y cantidad dificultan la separación de lo que es útil de lo que es superficial para el objetivo que desea alcanzar». La autora en su estudio afirma que el empresario necesita de orientación para saber cuáles son datos útiles para su empresa y cuáles pueden ser irrelevantes para la toma de decisiones y consecución de objetivos.

Oliveira, et al., 2016 indican que: «El proceso de globalización y los avances tecnológicos a lo largo de los siglos, hizo que la humanidad se adapte a nuevas necesidades». Con esa necesidad, el mercado exigió que se haga más de la mejor manera, en menor tiempoy con mayor precisión acarreando así algunas modificaciones relevantes en el mercado interno nacional y a partir del siglo XXI, con el extraordinario salto tecnológico de la era de la informática, la utilización de las computadoras aumentó aún más la competencia entre los mercados. Esos controles traídos al contexto actual, les dan a las micro y pequeñas empresas herramientas vitales para su continuidad.

Defaveri y Baldissera (2016) argumentan que la tecnología debe ser siempre aliada al proceso de gestión y que puede, también, volverse algo incómodo para los gestores de las MyPES. La contabilidad gerencial aparece como una herramienta para atender esa necesidad de organización, ayudando a las empresas a enfrentar los percances del proceso de gestión.

Con el crecimiento de los mercados, globalización y tecnología, se abrieron nuevas oportunidades para las MyPEs a nivel nacional. Esa apertura del mercado propició y fortaleció a las micro y pequeñas empresas; según SEBRAE (2014), en ese año las MyPES fueron las principales generadoras de riqueza en el sector comercial de Brasil, ya que respondieron por el $53.4 \%$ de PBI de este sector. Es así que aun teniendo esa enorme participación del mercado nacional las micro y pequeñas empresas terminan cerrando sus puertas incluso antes de alcanzar el tercer año de funcionamiento.

De acuerdo con el informe del SEBRAE (2016), fueron divulgadas las tasas de mortalidad de empresas de Brasil con un máximo de dos años de existencia y constituidas a partir de 2010, esa tasa era de 5\%. El mismo informe apunta que muchas razones justifican estos resultados, pero la principal causa es la falta de conocimiento sobre el mercado y el funcionamiento del negocio.

Esa falta de conocimiento del propio negocio, así como buscar la información necesaria para conocer el mercado se vuelve de suma importancia para el empresario que está iniciando su emprendimiento. No buscar esa información hace con que la empresa sufra las consecuencias de una administración que desconoce procedimientos administrativos que garanticen su existencia en el mercado. En este trabajo, se evidencia la forma como la contabilidad gerencial y sus herramientas pueden, junto a los sistemas de información, controles gerenciales y de desempeño de 
las empresas MyPES del rubro alimenticio, ayudar específicamente al empresario del sector del comercio minorista de alimentos, a tener mayor control y conocimiento de su actividad y la repercusión que proviene de una administración gerencial consciente de las informaciones encontradas.

Las empresas que actúan en el sector de comercio minorista de alimentos, encuadradas como micro y pequeñas empresas como restaurantes, bares y similares, pueden mejorar su desempeño alcanzando mejores resultados y performance económica al utilizar controles gerenciales y de desempeño, consiguiendo sus objetivos como organización y evidenciando resultados que promuevan su existencia, crecimiento y continuidad. En este contexto, se establece la siguiente pregunta de investigación: ¿Cuáles son las características de gestión, controles gerenciales y de desempeño de las empresas MyPES del rubro alimenticio? Y tomando como pauta esta interrogante, se tiene como alcance investigar las características de gestión, controles gerenciales y de desempeño de las empresas MyPEs del rubro alimenticio, y verificar su importancia para la continuidad de la entidad. Este estudio busca contribuir al uso de los controles gerenciales en MyPEs y su aplicación como herramienta de ayuda para la toma de decisiones repercutiendo en resultados financieros positivos.

\section{Metodología}

La metodología significa el camino, el trayecto, que en general puede ser reiniciado, cuando sea necesario (Menezes \& Silva, 2005). En este aspecto, la metodología desarrolla los procedimientos a ser usados en la construcción de la investigación. Esta investigación descriptiva fue realizada a partir de la investigación survey, levantamiento, con aplicación de cuestionarios enviados por e-mail para los gestores de las empresas en los meses de abril y mayo de 2017. Los gestores de diversos establecimientos fueron identificados por medio de una consulta al Sindicato de Hoteles, Restaurantes, Bares y similares de Florianópolis. A partir de esta selección, fue definida una población de 3563 empresas enmarcadas como Micro y Pequeñas empresas que actúan en el rubro de comercio minorista de alimentos, como bares y restaurantes. En total, 31 empresas respondieron al cuestionario. Así, la muestra incluyó empresas seleccionadas por accesibilidad. El instrumento de investigación consistió en un cuestionario dirigido a los gestores de las empresas medidos por la escala de Likert de 5 puntos. Las variables e informaciones fueron estructuradas conforme se evidencia en la tabla 1. 
Tabla 1

Elaboración del cuestionario

\begin{tabular}{|c|c|c|}
\hline Variables Explicativas & $\begin{array}{l}\text { Variables Explicativas } \\
\text { (indicadores) }\end{array}$ & Referencia \\
\hline $\begin{array}{l}\text { Planificación } \\
\text { (Plan de negocios) }\end{array}$ & $\begin{array}{l}\text { Desarrollo del plan de negocios } \\
\text { Desarrollo del plan de acción } \\
\text { Uso del plan de negocios }\end{array}$ & Feuser (2016) \\
\hline Controles Financieros & $\begin{array}{l}\text { Conocimiento del flujo de caja } \\
\text { Uso del flujo de caja } \\
\text { Uso de ingresos devengados }\end{array}$ & Feuser (2016) \\
\hline Factores de Marketing & $\begin{array}{l}\text { Investigación de mercados } \\
\text { Contacto con proveedores } \\
\text { Análisis del entorno }\end{array}$ & Feuser (2016) \\
\hline Control de Personal & $\begin{array}{l}\text { Control de acciones } \\
\text { Reclutamiento y selección } \\
\text { Entrenamiento }\end{array}$ & Feuser (2016) \\
\hline
\end{tabular}

Nota: Elaboración propia del equipo de investigación (2017).

En el cuestionario se verificó, en principio, la planificación que fue realizada para concretar el emprendimiento, la forma en que la información fue buscada por el empresario para fundamentar sus acciones. En la segunda variable, se verificó el conocimiento y uso del control financiero para ayudar al flujo de caja. En la tercera y cuarta variable, respectivamente, se verificó el contacto con los proveedores, la verificación del mercado, y el control del entrenamiento de funcionarios y colaboradores.
1. Análisis y Discusión de los resultados

1.1 Perfil de los encuestados

En la primera parte de la investigación, se buscó conocer los datos demográficos relacionados al género, año de nacimiento, año en que la empresa inició o fue adquirida, localización del establecimiento, cantidad de funcionarios al inicio y actualidad, además del nivel de educación de los gestores de esos establecimientos. 
Tabla 2

Perfil de los encuestados

\begin{tabular}{|c|c|c|c|}
\hline \multicolumn{2}{|c|}{ Género } & Año de Nacimiento & Frecuencia \\
\hline & & 1930 a 1940 & 1 \\
\hline \multirow[t]{2}{*}{ Femenino } & $3,23 \%$ & & \\
\hline & & 1941 a 1950 & 1 \\
\hline \multirow[t]{6}{*}{ Masculino } & $96,77 \%$ & & \\
\hline & & 1951 a 1960 & 1 \\
\hline & & 1961 a 1970 & 11 \\
\hline & & 1971 a 1980 & 12 \\
\hline & & 1981 a 1990 & 3 \\
\hline & & 1991 a 2000 & 2 \\
\hline
\end{tabular}

Cantidad de Funcionarios

\begin{tabular}{|c|c|c|c|c|}
\hline $\begin{array}{l}\text { Año de Inicio } \\
\text { de la Empresa }\end{array}$ & Frecuencia & \multicolumn{3}{|c|}{ Al inicio de Actualmente en la empresa } \\
\hline 1983 a 1992 & 3 & 1 a 5 & 10 a 14 & $25,80 \%$ \\
\hline 1993 a 2002 & 18 & 6 a 10 & 15 a 19 & $35,48 \%$ \\
\hline 2003 a 2012 & 9 & Yo y familia & 20 a 24 & $9,68 \%$ \\
\hline \multirow{2}{*}{$\begin{array}{c}2013 \text { en } \\
\text { adelante }\end{array}$} & 1 & Familiares & 25 a 29 & $9,68 \%$ \\
\hline & & & $\begin{array}{l}30 \text { a } 34 \\
35 \text { a } 39\end{array}$ & $\begin{array}{l}9,68 \% \\
9,68 \% \\
\end{array}$ \\
\hline
\end{tabular}

Nota: Elaboración propia del equipo de investigación (2017).

Se observa en la tabla 2, que la mayoría de los encuestados son de género masculino representados por el $96.77 \%$. La edad de los gestores está entre 36 y 45 años (nacidos entre 1971 y 1980) en 12 de los encuestados. Esto nuestra que los gestores pueden estar asumiendo los negocios de la familia. Se nota que esta característica termina por ayudar a la familia que ejerce la gestión, pues los jóvenes terminan especializándose más al participar de cursos y estudios universitarios. Defaveri y Baldissera (2016) en su estudio, apuntan que entre las MyPEs es común el control familiar, recayendo en la figura del gestor principal, la acción de control; los demás que también están encima de los funcionarios hacen parte de la familia del gestor, lo que puede explicar que las empresas investigadas posean más de un socio. 
La cantidad de funcionarios también es un dato importante levantado en este estudio. Esta comparación, evidencia la evolución del cuadro de funcionarios y confirma que, para que una empresa crezca, necesita de más colaboradores con metas y acciones alineadas a la organización.

Al inicio de su actuación, las empresas poseían principalmente de 1 a 5 funcionarios como se puede verificar con el $54.84 \%$ de los encuestados. Se observa que el $35.48 \%$ de las organizaciones poseen entre 15 y 19 funcionarios; $25.80 \%$, entre 10 y 14 , y las demás suman el 9.68\%. La justificación para el aumento de funcionarios puede ser atribuida al uso correcto de los controles gerenciales que permiten una buena gestión y acompañamiento de las acciones de toda la organización, reflejando así un resultado económico mayor que al mismo tiempo permite la contratación de más funcionarios.

Este trabajo también muestra el nivel de instrucción de los gestores. Ese dato puede influenciar en la toma de decisiones, haciendo que la información que ya es conocida puede hacer la diferencia en los negocios. Conforme se evidencia en la tabla 3.

Tabla 3

Nivel de Educación completa y área de la carrera superior/técnica

\begin{tabular}{llll}
\hline \multicolumn{1}{c}{ Estudios completos } & \multicolumn{2}{l}{ Área de formación - especialización } \\
\hline Primaria & $3.23 \%$ & No tiene & $64.52 \%$ \\
Secundaria & $61.29 \%$ & Administración & $12.90 \%$ \\
Superior & $32.26 \%$ & Derecho & $9.68 \%$ \\
Post - graduación & $3.23 \%$ & Contabilidad & $3.23 \%$ \\
& & Finanzas & $3.23 \%$ \\
& & Corporativas & $3.23 \%$ \\
& & Ingeniería & $3.23 \%$ \\
\hline
\end{tabular}

Nota: Elaboración propia del equipo de investigación (2017).

La secundaria representa el mayor porcentaje con $61.29 \%$ de los gestores teniendo tal formación. Con 32.26\% los estudios superiores poseen la segunda mayor cantidad de gestores encuestados que pueden tener profesionales que ya terminaron la graduación. El 3.23\% culminó la primaria y la pos-graduación con el mismo porcentaje, se puede verificar que apenas una pequeña parte de los gestores que terminar sus estudios superiores, buscan un pos-grado con el objetivo de mejorar su conocimiento.

Se observa que el $64.52 \%$ de los gestores no tienen estudios superiores o técnicos en alguna actividad; $\mathbf{1 2 . 9 0 \% ~ s o n ~ p r o v e n i e n - ~}$ tes de cursos de administración; seguidos de $9.68 \%$ con estudios en Derecho (como segunda mayor formación), y los demás suman el 3.23\% y corresponden a Economía, Contabilidad, Finanzas, 
Corporativas e Ingeniería. La búsqueda por el espíritu emprendedor puede ser el factor que lleva a los gestores a buscar un mercado alternativo para colocar en práctica parte de aquello que aprendieron en sus cursos superiores.

\subsection{Análisis en cuanto a los aspectos de control}

Las organizaciones utilizan los controles gerenciales para definir sus estrategias de negocio, su planificación, el control financiero de la organización, el acompañamiento del mercado por medio del marketing, el alineamiento de los funcionarios en cuanto a sus objetivos, en relación a los de la organización, y qué ocurre con el desempeño de la organización cuando estos controles son aplicados, pudiendo reflejarse ello en el resultado económico.

El primer levantamiento trata sobre la estrategia del negocio, se buscó conocer los objetivos de la organización, cómo los gestores iniciaron la busca de informaciones para la apertura o adquisición de sus negocios, cuáles fueron los objetivos y medios de acción para alcanzarlos. Conforme se muestra en la tabla 4.

Tabla 4

Estrategia del negocio

\begin{tabular}{lcc}
\hline & Sí & No \\
\hline $\begin{array}{l}\text { ¿Los objetivos de su empresa están } \\
\text { claros y definidos? }\end{array}$ & $83,87 \%$ & $16,13 \%$ \\
\hline $\begin{array}{l}\text { ¿xisten procedimientos precisos } \\
\text { para alcanzar los objetivos estraté- } \\
\text { gicos y para encontrar soluciones } \\
\text { para los problemas estratégicos? }\end{array}$ & $35,48 \%$ & $64,52 \%$ \\
\hline
\end{tabular}

Nota: Elaboración propia del equipo de investigación (2017).

Para el $83.87 \%$ de los encuestados, los objetivos son claros y definidos, en oposición al $16.13 \%$ que creen que no tienen un objetivo claro y definido de su emprendimiento. Este resultado evidencia que los gestores se preocupan inicialmente con sus organizaciones, teniendo como objetivo un posicionamiento económico y social ideal para su planificación y que automáticamente tienen un retorno financiero esperado para su planificación de vida profesional y personal. Para el $35.48 \%$ de los encuestados, sus organizaciones poseen tal control de acompañamiento y solución de problemas que actúa de forma precisa; sin embargo, para el $64.52 \%$, los controles no son precisos, o están ausentes. Se puede observar que la mayoría de los empresarios y los gestores poseen objetivos definidos claramente para su organización; sin embargo, no tienen controles ni procedimientos estratégicos para que la organización encuentre soluciones para los problemas y alcance sus objetivos. 
Este mismo resultado es percibido en el al mismo tiempo, permiten al gestor realiestudio de Defaveri y Baldissera (2016), zar evaluaciones sobre la evolución de su donde aunque existen controles defini- empresa, permitiendo que puedan tomarse dos, existen dificultades que impiden el acciones para alcanzar el objetivo. En lo buen funcionamiento de la organización referente a la planificación, los resultacon el uso de controles precisos. En el dos evidenciaron el nivel de búsqueda de estudio de Lima e Imoniana (2008), se conocimiento sobre el emprendimiento y listan las herramientas de planificación, cuál es la forma más utilizada para buscar controles gerenciales como controles de información. Los resultados son presenpresupuesto, stock, ventas y costos. Estos, tados en la tabla 5 .

Tabla 5

Proceso de planificación

\begin{tabular}{|c|c|c|c|c|c|c|c|}
\hline & Nunca & Raramente & $\begin{array}{c}\text { Algunas } \\
\text { veces }\end{array}$ & $\begin{array}{c}\text { Casi } \\
\text { Siempre }\end{array}$ & Siempre & Promedio & $\begin{array}{l}\text { Desviación } \\
\text { Estándar }\end{array}$ \\
\hline $\begin{array}{l}\text { ¿Usted buscó infor- } \\
\text { mación o compartió } \\
\text { experiencias con } \\
\text { familiares o amigos } \\
\text { que trabajan en el } \\
\text { mismo rubro? }\end{array}$ & $9,68 \%$ & $25,81 \%$ & $38,70 \%$ & $25,81 \%$ & $\mathrm{o} \%$ & $18,71 \%$ & 0,15 \\
\hline $\begin{array}{l}\text { En relación al nivel } \\
\text { de asociación, ¿usted } \\
\text { participó o participa } \\
\text { de acciones en } \\
\text { conjunto con otras } \\
\text { empresas (compras, } \\
\text { cooperativas, asocia- } \\
\text { ciones empresariales } \\
\text { y redes sectoriales) } \\
\text { del mismo rubro? }\end{array}$ & $22,58 \%$ & $25,81 \%$ & $38,71 \%$ & $12,90 \%$ & $\mathrm{o} \%$ & $16,13 \%$ & 0,14 \\
\hline $\begin{array}{l}\text { ¿Con qué frecuencia } \\
\text { usted utilizo o utiliza } \\
\text { servicios especiali- } \\
\text { zados, como Sebrae, } \\
\text { Universidades, } \\
\text { Consultorías, etc.? }\end{array}$ & $9,68 \%$ & $16,12 \%$ & $32,27 \%$ & $25,81 \%$ & $16,12 \%$ & $21,50 \%$ & 0,09 \\
\hline
\end{tabular}

Nota: Elaboración propia del equipo de investigación (2017). 
Cuando se les preguntó en relación a la búsqueda de información de hechos que sucedieron a miembros de la familia o amigos que trabajan en el mismo rubro, 9.68\% de los encuestados afirmaron que no fue necesario hacerla, o nunca fue realizada. Para el $25.81 \%$ sucedieron raramente. El resultado puede estar en concordancia con el estudio de Dos Santos et al., 2016, en donde, para la mayoría de los encuestados, el empresario toma sus decisiones basado en su propio conocimiento y experiencia, lo que descarta la búsqueda de fuentes externas como familiar, amigos o personas relacionadas que trabajan en el mismo rubro de actividad.

Para la segunda pregunta, cerca del $12.90 \%$ de los encuestados respondieron que buscan con mayor frecuencia participar en conjunto con otras empresas, cooperativas, asociaciones o similares. Para el 22.58\% de los gestores consultados esa búsqueda no ocurrió hasta el momento, o no fue necesaria.

La información descrita anteriormente, complementa directamente la tercera pregunta en donde se verifica la frecuencia de los encuestados en buscar y participar de servicios especializados como SEBRAE, universidades, consultorías y otras formas de obtener información sobre el rubro del negocio, donde el $32.27 \%$ de los encuestados dijeron buscar algunas veces estas entidades especializadas para realizar consultas. También, con $25.81 \%$ las empresas casi siempre buscan por medio de servicios especializados como los ofrecidos por SEBRAE, obtener herramientas y formas de gestión que permitan controlar mejor su empresa, cuando la cuestión es la planificación.

El tercer levantamiento apuntado en este estudio es sobre el control financiero. Con él, la organización identifica sus objetivos a corto, mediano y largo plazo, se puede planear la caja para los mejores días de compras y pago de cuentas. Se identifica las transacciones ocurridas tanto en las entradas de los recursos como en las salidas para compras de materiales y mercadería. Conforme se puede evidenciar en la tabla 6. 


\section{Tabla 6}

Control financiero

\begin{tabular}{|c|c|c|c|c|c|c|c|}
\hline & Nunca & Raramente & $\begin{array}{c}\text { Algunas } \\
\text { Veces }\end{array}$ & $\begin{array}{c}\text { Casi } \\
\text { Siempre }\end{array}$ & Siempre & Promedio & $\begin{array}{l}\text { Desviación } \\
\text { Estándar }\end{array}$ \\
\hline $\begin{array}{l}\text { ¿Usted acos- } \\
\text { tumbra hacer un } \\
\text { plan de acción } \\
\text { para alcanzar } \\
\text { las metas de la } \\
\text { empresa? }\end{array}$ & o\% & o\% & $22,58 \%$ & $48,39 \%$ & $29,03 \%$ & $27,10 \%$ & 0,21 \\
\hline $\begin{array}{l}\text { ¿Usted utiliza } \\
\text { esta planifi- } \\
\text { cación para } \\
\text { acompañary } \\
\text { controla las } \\
\text { acciones y tomar } \\
\text { decisiones? }\end{array}$ & o\% & $6,45 \%$ & $16,13 \%$ & $54,84 \%$ & $22,58 \%$ & $26,24 \%$ & 0,21 \\
\hline $\begin{array}{l}\text { En relación al } \\
\text { control finan- } \\
\text { ciero, ¿usted } \\
\text { conoce con deta- } \\
\text { lle las entradas } \\
\text { y salidas de caja } \\
\text { que ocurrirán en } \\
\text { el mes? }\end{array}$ & o\% & o\% & $12,90 \%$ & $48,39 \%$ & $38,71 \%$ & $28,38 \%$ & 0,22 \\
\hline $\begin{array}{l}\text { ¿Usted utiliza } \\
\text { el flujo de caja } \\
\text { para controlar } \\
\text { las entradas y } \\
\text { salidas de cajá de } \\
\text { su empresa? }\end{array}$ & o\% & $3,23 \%$ & $38,71 \%$ & $35,48 \%$ & $22,58 \%$ & $25,16 \%$ & 0,18 \\
\hline
\end{tabular}

Nota: Elaboración propia del equipo de investigación (2017).

Se observa que el $48.39 \%$ de los gestores casi siempre buscan hacer planes de acción para alcanzar las metas de la empresa. Para el 29.03\% de los gestores, este plan de acción es realizado siempre por el propio gestor, o sea, no depende de un tercero designado o subordinado al empresario.
La segunda pregunta aduce, para el control financiero que $54.84 \%$ de los gestores de las empresas casi siempre usan la planificación para acompañar y controlar las decisiones, $\mathbf{2 2 . 5 8 \%}$ siempre usan la planificación, $16.13 \%$ lo hacen algunas veces y $6.45 \%$ raramente. 
En relación a las salidas de caja con conocimiento de los gestores, la tercera pregunta buscó verificar cómo dentro de la muestra los gestores conocen las entradas y salidas de caja de sus empresas. Con $48.39 \%$ de los encuestados teniendo conocimiento sobre casi todas las salidas de caja, en segundo lugar, aparece un $38.71 \%$ con total seguridad sobre el flujo de caja. Ese control puede ser adaptado conforme la necesidad del gestor, evidenciando detalles sobre las salidas y entradas de su flujo de caja.
El cuarto levantamiento realizado en este trabajo es relacionado a los factores de marketing. En él se identifica si la búsqueda de información del mercado servirá para que los clientes disfruten de los productos de esa empresa, cómo los gestores actuaron para buscar esa información. Este procedimiento busca prever qué acciones puede hacer la diferencia en el mercado, conforme a la tabla 7.

Tabla 7

Factores de marketing

\begin{tabular}{|c|c|c|c|c|c|c|c|}
\hline & Nunca & Raramente & $\begin{array}{c}\text { Algunas } \\
\text { Veces }\end{array}$ & $\begin{array}{c}\text { Casi } \\
\text { Siempre }\end{array}$ & Siempre & Promedio & $\begin{array}{c}\text { Desviación } \\
\text { Estándar }\end{array}$ \\
\hline $\begin{array}{l}\text { Antes de abrir } \\
\text { la empresa, } \\
\text { ¿usted investigo } \\
\text { el mercado de } \\
\text { acción y el perfil } \\
\text { de los clientes? }\end{array}$ & о\% & о\% & $25,81 \%$ & $58,06 \%$ & $16,13 \%$ & $26,02 \%$ & 0,24 \\
\hline $\begin{array}{l}\text { Para abrir la } \\
\text { empresa, ¿usted } \\
\text { identifico o hizo } \\
\text { contacto con } \\
\text { sus principales } \\
\text { proveedores? }\end{array}$ & о\% & о\% & $9,68 \%$ & $61,29 \%$ & $29,03 \%$ & $27,96 \%$ & 0,26 \\
\hline $\begin{array}{l}\text { Antes de comen- } \\
\text { zar la empresa, } \\
\text { ¿usted identi- } \\
\text { fico y analizó a } \\
\text { sus principales } \\
\text { competidores } \\
\text { precios, aten- } \\
\text { ción, competiti- } \\
\text { vidad, etc.? }\end{array}$ & о\% & о\% & $25,81 \%$ & $45,16 \%$ & $29,03 \%$ & $26,88 \%$ & 0,20 \\
\hline
\end{tabular}

Nota: Elaboración propia del equipo de investigación (2017). 
Se nota que el $59.06 \%$ de los investigados casi siempre intervinieron el mercado de accióny el perfil del cliente. Esto es importante pues no vale la pena instalar un establecimiento con productos sofisticados en locales en los cuales el público no tiene poder de compra. Seguido por un $25.81 \%$ que investigaron algunas veces el mercado y el perfil de sus clientes, $16.13 \%$ tuvieron cuidado e investigaron en todos los aspectos el mercado y el perfil del cliente.

La segunda pregunta identifica queel $61.29 \%$ de las empresas casi siempre identificaron y contactaron a sus principales proveedores incluso antes de abrir la empresa; 29.03\% lo hicieron asiduamente, es decir, siempre. El mayor porcentaje puede estar vinculado a empresas como restaurantes comunes en donde no existe un producto exclusivo de un solo proveedor, lo que permite la comparación de precios entre los principales productos de un establecimiento del rubro alimenticio.
La tercera pregunta permitió observar se las empresas analizan a sus competidores, práctica de precios, atención, competitividad del mercado. Con $45.16 \%$ casi siempre las empresas lo hacen; $29.03 \%$ siempre; $25.81 \%$ algunas veces. Estos porcentajes puede tener influencia en el tipo de establecimiento alimenticio que está siendo planeado, como una franquicia, en que todo es establecido con anterioridad durante la propuesta, incluso hasta el local del establecimiento que muchas veces no es elegido por el propietario del negocio.

El quinto levantamiento identifica a los encuestados en cuando a las formas de buscar colaboradores para la organización, si estos ya tienen experiencia en el mercado, se tienen metas alineadas con la organización y si reciben entrenamiento para tener atención personalizada y volcada a los objetivos de la organización. Conforme se muestra en la tabla 8 , sobre el control del personal. 


\section{Tabla 8}

Control de personal

\begin{tabular}{|c|c|c|c|c|c|c|c|}
\hline & Nunca & Raramente & $\begin{array}{c}\text { Algunas } \\
\text { Veces }\end{array}$ & $\begin{array}{c}\text { Casi } \\
\text { Siempre }\end{array}$ & Siempre & Promedio & $\begin{array}{c}\text { Desviación } \\
\text { Estándar }\end{array}$ \\
\hline $\begin{array}{l}\text { En relación a } \\
\text { los colabora- } \\
\text { dores, ¿usted } \\
\text { controla y } \\
\text { verifica si las } \\
\text { acciones de } \\
\text { sus funcio- } \\
\text { narios están } \\
\text { alineadas } \\
\text { con las } \\
\text { metas de la } \\
\text { empresa? }\end{array}$ & o\% & O\% & $29,03 \%$ & $45,16 \%$ & $25,81 \%$ & $26,45 \%$ & 0,20 \\
\hline $\begin{array}{l}\text { ¿Usted } \\
\text { intenta } \\
\text { reclutar y } \\
\text { seleccionar } \\
\text { los funciona- } \\
\text { rios antes de } \\
\text { contratarlos? }\end{array}$ & o\% & $3,23 \%$ & $29,03 \%$ & $48,39 \%$ & $19,35 \%$ & $25,59 \%$ & 0,20 \\
\hline $\begin{array}{l}\text { ¿Usted } \\
\text { entrena a sus } \\
\text { funcionarios? }\end{array}$ & o\% & o\% & $6,45 \%$ & $32,26 \%$ & $61,29 \%$ & $30,32 \%$ & 0,27 \\
\hline
\end{tabular}

Nota: Elaboración propia del equipo de investigación (2017).

Se observa que el $45.16 \%$ casi siempre posee control sobre las acciones de sus subordinados, $29.03 \%$ lo hacen algunas veces y el $25.81 \%$ siempre acompaña y controla sus funcionarios para tener seguridad de que están de acuerdo con los objetivos de la organización.

De esto se desprende que el $48.39 \%$ de los encuestados casi siempre usa el reclutamiento como forma de ganar colaboradores alineados a su negocio; $29.03 \%$ lo hace algunas veces, el 19.35\% siempre lo hace y el 3.23\% raramente lo hace directamente. Existen empresas de base de datos en las que el trabajador puede colocar su currículum vitae a disposición de las empresas para una pre-selección, siendo esta una forma más fácil de filtrar trabajadores que están siendo necesitados por la empresa, lo que directamente no puede ser considerado como reclutamiento. Además, 61.29\% siempre entrenan a sus colaboradores, seguido de un $32.26 \%$ que lo hacen casi siempre y luego de un $6.45 \%$ que lo hacen algunas veces. 
El desempeño de las organizaciones fue financiero sobre las ventas y entradas de el sexto levantamiento evaluado en este la empresa, como el flujo de caja, toda la trabajo. En esta parte se preguntó a los gestores en relación al desempeño que la empresa tiene a lo largo de su existencia. Los resultados en conjunto que todas las acciones de las preguntas anteriores pueden generar para la empresa, toda la preparación para el conocimiento del mercado, sobre la forma deactuar, planificación, conocimiento preparación del personal y entrenamiento de los mismos generan resultados como el crecimiento de la organización, aumento de ventas y consecuentemente aumento del lucro, teniendo como consecuencia, el aumento del prestigio social de la empresa en la sociedad en que se ha establecido. Los resultados son presentados en la tabla 9 .

Tabla 9

Resultados sobre el desempeño

\begin{tabular}{|c|c|c|c|c|c|c|c|}
\hline & Nunca & Raramente & $\begin{array}{c}\text { Algunas } \\
\text { Veces }\end{array}$ & $\begin{array}{c}\text { Casi } \\
\text { Siempre }\end{array}$ & Siempre & Promedio & $\begin{array}{c}\text { Desviación } \\
\text { Estándar }\end{array}$ \\
\hline ¿Usted percibe que & & & & & & & \\
\hline $\begin{array}{l}\text { las ventas de su } \\
\text { empresa tuvieron un } \\
\text { crecimiento desde el } \\
\text { inicio? }\end{array}$ & $\mathrm{o} \%$ & $3,23 \%$ & $9,68 \%$ & $64,52 \%$ & $22,58 \%$ & $27,10 \%$ & 0,26 \\
\hline $\begin{array}{l}\text { ¿Usted percibe que el } \\
\text { lucro de su empresa } \\
\text { tuvo un crecimiento } \\
\text { desde el inicio? }\end{array}$ & $\mathrm{O} \%$ & o\% & $22,58 \%$ & $64,52 \%$ & $12,90 \%$ & $26,02 \%$ & 0,27 \\
\hline $\begin{array}{l}\text { ¿Usted percibe que el } \\
\text { número de funcio- } \\
\text { narios de su empresa } \\
\text { tuvo un crecimiento } \\
\text { desde el inicio? }\end{array}$ & o\% & o\% & $22,58 \%$ & $61,29 \%$ & $16,13 \%$ & $26,24 \%$ & 0,25 \\
\hline $\begin{array}{l}\text { ¿Usted percebe que la } \\
\text { imagen y el prestigio } \\
\text { social de su empresa } \\
\text { tuvo un crecimiento } \\
\text { desde el inicio? }\end{array}$ & o\% & o\% & $6,45 \%$ & $74,19 \%$ & $19,35 \%$ & $27,52 \%$ & 0,31 \\
\hline
\end{tabular}

Nota: Elaboración propia del equipo de investigación (2017).

Se observa que el $64.52 \%$ respondió que casi siempre percibe el aumento en las ventas del establecimiento; $22.58 \%$ siempre percibe aumento en las ventas, seguidos por un $9.68 \%$ de establecimientos que algunas veces percibe que el volumen de ventas sube y el $3.23 \%$ que raramente percibe que su volumen de ventas aumenta. Se verifica que la tabla está de acuerdo con el estudio de Feuser(2016) en relación a que cuando 
se aplican como herramientas de gestión, los controles gerenciales promueven que la empresa aumente su productividad, capacidad de atención, aumento de contratación de funcionarios y directamente el aumento de facturación y lucro. La autora afirma, además, que el capital humano es extremadamente importante y determinante en la consecución de buenos resultados.

Para aproximadamente el 61\% de los encuestados, hubo un aumento significativo de colaboradores, para el $22.58 \%$, algunas veces y para el $16.13 \%$ ese aumento es percibido como de mayor intensidad. El séptimo levantamiento verifica la cuestión del control en las organizaciones. El uso de controles permiteal gestor atribuir ajustes que pueden ser esenciales para alcanzar resultados diferenciados en el mercado. Los controles tienen por objetivo también mostrar si los resultados que antes fueron planeados están siendo obtenidos. La tabla 10 presenta los resultados encontrados.

\section{Tabla 10}

\section{Resultados sobre el control}

\begin{tabular}{|c|c|c|c|c|c|c|c|}
\hline & Nunca & Raramente & $\begin{array}{c}\text { Algunas } \\
\text { Veces }\end{array}$ & $\begin{array}{c}\text { Casi } \\
\text { Siempre }\end{array}$ & Siempre & Promedio & $\begin{array}{c}\text { Desviación } \\
\text { Estándar }\end{array}$ \\
\hline $\begin{array}{l}\text { En su percepción como } \\
\text { propietario o gestor de }\end{array}$ & & & & & & & \\
\hline $\begin{array}{l}\text { esa empresa, ¿siente } \\
\text { falta (o ve importan- } \\
\text { cia) en la implemen- } \\
\text { tación de que tipos de } \\
\text { controles? }\end{array}$ & o\% & $3,23 \%$ & $22,58 \%$ & $51,61 \%$ & $22,58 \%$ & $26,24 \%$ & 0,19 \\
\hline
\end{tabular}

Nota: Elaboración propia del equipo de investigación (2017).

En la percepción de los encuestados, 51.61\% casi siempre sienten la falta u observan la importancia de utilizar controles gerenciales en sus establecimientos. Ya las empresas que siempre o algunas veces perciben la importancia de los controles están igualadas y apenas un $3.23 \%$ de los encuestados raramente sienten la falta u observan la importancia de los controles en sus emprendimientos.

Los resultados presentados en las tablas anteriores muestran que la mayoría de empresas siente necesidad de la existencia de controles gerenciales y que para obtener los resultados que planearon anteriormente son necesarias acciones no solo de los gestores, sino de todo su equipo de funcionarios y colaboradores. Externamente, los proveedores y demás contactos de la organización también influyen en la planificación al consultar un especialista en planificación, sea financiero o personal.

Así, como en el estudio de Defaveri y Baldissera (2016), por los resultados que se obtuvo con el estudio, se evidencia que los gestores de las empresas buscan verificar la planificación de la organización y las metas previamente trazadas para obtener 
y controlar los resultados que están siendo obtenidos.

\section{Consideraciones finales}

El estudio tuvo como objetivo verificar las características de gestión, controles gerenciales y de desempeño de las empresas MyPEs del rubro alimenticio, y verificar la continuidad de esos establecimientos. Los resultados evidenciaron que el perfil promedio de las empresas consiste en un emprendimiento que posee objetivos claros y definidos, y que está apto para utilizar procedimiento que pueden trabajar a favor de sus objetivos.

Se verifica que la mayoría de los gestores buscan informaciones en fuentes próximas como familiares o amigos que trabajan en el mismo rubro o actividad, que buscan orientarse por medio de servicios especializados y consultorías direccionadas a su emprendimiento, y que también se comunican con otras empresas del rubro por medio de eventos organizados por órganos que los representan, formalizando convenios que les traen mayores beneficios corroborando resultados positivos. Los resultados de este estudio convergen con el estudio de Feuser (2016) en el cual fue verificado que para que la organización tenga resultados positivos, es necesaria la implantación de controles gerenciales e interpretar de manera correcta los resultados obtenidos siendo estos comparados con lo planificado.

Los resultados refuerzan que la mayoría de los gestores acostumbran realizar un plan de acción para sus empresas a fin de verificar el alcance de metas y acompañar la evolución por medio de controles que pueden, a medida de lo posible, evidenciar las entradas y salidas de caja para que la planificación no tenga "huecos", o sea, que puedan causar problemas de flujo de caja o pagos que no estaban planificados anteriormente.

Se verifica que el control de personal es visto por los gestores como una herramienta que puedealinear los objetivos de la organización con sus funcionarios, lo que permite una comunicación más clara y objetiva dentro de la empresa. La necesidad de entrenamiento y reclutamiento de profesionales que van a atender las necesidades del público objetivo de establecimiento con el objetivo de mostrar al mercado el diferencial de la empresa.

Finalmente, la aplicación de los controles gerenciales en las MyPEs, evidenció un aumento en la recaudación y lucro. Hubo también un aumento de contratación, permitiendo de esta manera expandir la atención a un número mayor de clientes. La cantidad de funcionarios se mostró mucho mayor que al inicio de las empresas, lo que significa que estas organizaciones consiguen administrar sus colaboradores y alcanzar los objetivos que fueron planificados anteriormente.

Se concluye que los controles gerenciales ayudan de manera positiva al gestor en la administración de su negocio. Se nota lo mismo aplicando controles gerenciales, las empresas aun desean tener intervenciones que supuestamente puedan contribuir aún más en sus procesos, dejándolos con mayor comprensión de los procedimientos en todos los niveles de la organización.

De esta forma, las empresas estudiadas usan controles gerenciales para controlar sus 
objetivos y acciones, buscando la mejora de sus procesos que los llevan a alcanzar sus objetivos, donde los controles son de total importancia para la toma de decisiones en todos los aspectos de la organización, desde el inicio de la empresa, en su fase inicial de planificación, en cuantoa mantenimientoy administración del negocio para perpetuarse en la actividad económica, generando así una contribución económica y social para la ciudad de Florianópolis - Santa Catarina (Brasil). Este trabajo también incentiva la investigación de trabajos futuros, con el objetivo de acompañar la evolución de las micro y pequeñas empresas, que, en su conjunto, fortalecen la economía del país, así como ampliar la muestra a otras regiones para poder realizar un estudio comparativo.

\section{Referencias}

Anthony, R.N.; \& Govindarajan, V. (2001). "Sistemas de controle gerencial". São Paulo: Atlas.

Antonik, L. R. (2004). Administração financeira das pequenas e médias empresas: ferramentas financeiras simples auxiliam na gestão da empresa e orientam a tomada de decisões. Revista FAE Business, 8, 35-39.

Beuren, I. M. (2010). "Como Elaborar Trabalhos Monográficos em Contabilidade: Teoria e Prática”. São Paulo: Atlas.

Boaventura, W. R. (2003). "A Contabilidade Gerencial e a micro e pequena empresa: estudo de um sistema de registros e informações”. Mestrado em Controladoria e Contabilidade Estratégica. Unifecap, São Paulo, Brasil.

Brasil (2006). "Lei Complementar 123, de 14 de dezembro de 2006. Institui o Estatuto Nacional da Microempresa e da Empresa de Pequeno Porte”. Diário Oficial da União. Brasília.

Brasil (2016). "Lei Complementar 10.406, de 10 de janeiro de 2002". Disponível em: http://www.planalto.gov.br/ccivil_03/ leis/2002/L10406.htm. Acesso em: 05/04/2016.

Campos, B. R.; \& Gáudio, A. (2015). A utilização de ferramentas de controle gerencial em micro e pequenas empresas da Região Metropolitana do Rio de Janeiro. "Revista da Micro e Pequena Empresa", 8(3), 66-78.

Cooper, D. R.; \& Schindler, P. S. (2003). "Métodos de pesquisa em administração”. Porto Alegre: Bookman.

Defaveri, I. R.; \& Baldissera, J. F. (2016). “A influência dos sistemas de controle gerencial na tomada de decisão dos gestores de MPEs”. X Congresso Anpcont, Ribeirão Preto. São Paulo.

Dos Santos, V., Dorow, D. R.; Beuren, I. M. (2016). Práticas gerenciais de micro e pequenas empresas. "Revista Ambiente Contábil”, 8(1), 164.

Feuser, H.O.L. (2016). Desempenho de micro e pequenas empresas: a relação entre o controle gerencial e as características do empreendedor. Dissertação de 
Mestrado (Contabilidade) Universidade Federal do Paraná, Curitiba, Brasil.

Figueiredo, A.; \& Caggiano, P. C. (2004). “Controladoria. Teoria e Pratica”. São Paulo: Atlas.

IBGE - Instituto Brasileiro de Geografia e Estatística. (2003). "As micro e pequenas empresas comerciais e de serviços no Brasil: 2001”. IBGE, Brasil.

Leone, N.M.C.P.G. (1999). "As especificidades das pequenas e médias empresas”. Revista de Administração da Universidade de São Paulo, 34(2), 91-94.

Lima, A. N.; \& Imoniana, J. O. (2008). Um estudo sobre a importância do uso das ferramentas de controle gerencial nas micro, pequenas e médias empresas industriais no Município de São Caetano do Sul. "Revista da Micro e Pequena Empresa”, 2(3), 28-48.

Pinheiro, M. (1996). “Gestão e desempenho das empresas de pequeno porte”. São Paulo. Tese (Doutorado). Departamento de Administração, Faculdade de Economia e Administração, Universidade de São Paulo, São Paulo, Brasil.

Marion, J. C. (2009). "Contabilidade Empresarial”. São Paulo: Atlas.

Martins, G. A.; \& Theóphilo, C. R. (2007). "Metodologia da Investigação Científica para Ciências Sociais Aplicadas”. São Paulo: Atlas.
Menezes, E. M.; Silva, E. L. (2001). "Metodologia da pesquisa e elaboração de dissertação". Florianópolis: Laboratório de ensino a distância da UFSC.

Oliveira, A. G., Müller, A. N. \& Nakamura, W. T. (2000). A utilização das informações geradas pelo sistema de informação contábil como subsidio aos processos administrativos nas pequenas empresas. "Revista FAE”, 3(3), 1-12.

Padoveze, C. L. (2012). "Contabilidade Gerencial”. Curitiba: IESDE Brasil.

Padoveze, C. L. (2010). “Contabilidade Gerencial: um enfoque em sistema de informação contábil”. São Paulo: Atlas.

Prodanov, C. C.; \& Freitas, E. C. (2009). "Metodologia do trabalho científico". Novo Hamburgo: Feevale.

Richardson, R. J. (1999). "Pesquisa social: métodos e técnicas”. São Paulo: Atlas.

Ruengdet, K.; \& Wongsurawat, W. (2010). Characteristics of successful small and micro community enterprises in rural Thailand. "Journal of Agricultural Education and Extension”, 16(4), 385-397.

SEBRAE - Serviço Brasileiro de Apoio às Micro e Pequenas Empresas (2013). Disponível em: https://www.sebrae. com.br/Sebrae/Portal\%2oSebrae/ Anexos/Sobrevivencia_das_empresas_n o_Brasil=2013.pdf . Acesso em: 25/03/2017. 
SEBRAE - Serviço Brasileiro de Apoio às Micro e Pequenas Empresas (2014). Disponível em:https://www.sebrae. com.br/sites/PortalSebrae/ufs/mt/ noticias/micro-e-pequenas-empresas- geram-27-do-pib-do-brasil,adofc70646467410VgnVCM2000003c74010aRCRD . Acesso em 23/03/2017.

SEBRAE - Serviço Brasileiro de Apoio às Micro e Pequenas Empresas (2011). Disponível emhttps://www.sebrae.com. br/Sebrae/Portal\%2oSebrae/Anexos/ Sobrevivencia_das_empresas_no_ Brasil_2011.pdf. Acesso em 27/03/2017.

SEBRAE - Serviço Brasileiro de Apoio às Micro e Pequenas Empresas (2013). Disponível em <https://www.sebrae. com.br/Sebrae/Portal\%2oSebrae/ Anexos/Relatorio\%2oEstadual.pdf>

SEBRAE - Serviço Brasileiro de Apoio às Micro e Pequenas Empresas (2014). Disponível emhttps://www.sebrae. com.br/Sebrae/Portal\%2oSebrae/ Estudos\%20e\%2oPesquisas/Participa cao\%2odas\%2omicro\%2oe\%2opequenas\%2oempresas.pdf . Acesso em 27/03/2017.

SEBRAE - Serviço Brasileiro de Apoio às Micro e Pequenas Empresas (2013). Disponível emhttp://www.sebrae. c2q om.br/Sebrae/Portal\%2o Sebrae/Anexos/Anuario\%2odo\%20 Trabalho\%20Na\%2oMicro\%2oe\%20 Pequena\%2oEmpresa_2013.pdf. Acesso em 27/03/2017.

Silva, D.; \& Postiglione, E. M. (2016). “Uso Efetivo de Controles Internos em uma Microempresa de Santa Maria: Fundamento Para o Crescimento e a Manutenção do Negócio”. 1 $^{\circ}$ Congresso de Contabilidade da UFRGS. Universidade Universidade Federal do Rio Grande do Sul, Porto Alegre, Brasil.

Villa, P. (2012). —O monólogo contábil: uma análise do uso da contabilidade gerencial em micro e pequenas empresasà luz do processo comunicacional segundo Bakhtin”. Dissertação de Mestrado. UFPR, Curitiba, Brasil. 